\title{
Research on College English TPACK Framework Teaching from the Perspective of Connectionism
}

\author{
Xiuzhen Xie \\ A1103, Hongdu Yuan, Ziwei Rd, Xinhua Town, Huadu District, Guangzhou ,Guangdong Province, China
}

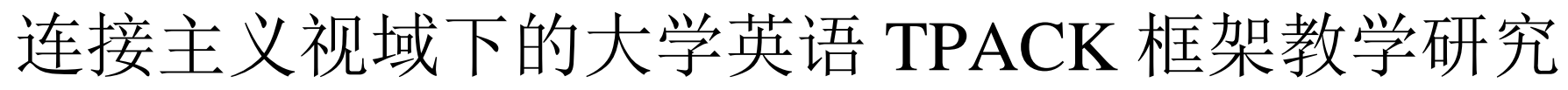

谢秀珍

广州工商学院, 广州, 中国

\begin{abstract}
The rapid development of information technology, international and domestic education reform and development trends request new requirements for University English teaching, We must use modern educational technology equipment to promote college English teaching. The implementation of University English information teaching is not only in line with the needs of China's economic development and international exchange, but also in line with the Uuniversity English course teaching requirements." From the perspective of connection theory, this paper proposes the construction and implementation of college English teaching under the framework of TPACK which based on the theory of connectivism.
\end{abstract}

Keywords-Connectivism; TPACK; College English teaching

摘要一信息技术的飞速发展, 国际, 国内教育改革发展趋势 对大学英语教学提出了新的要求, 用现代教育技术装备推动大学 英语教学, 推行大学英语信息化教学, 既符合我国经济发展和国 际交流的需要, 也符合《大学英语课程教学要求》, 本文从连接主 义理论视角, 针对大学英语教学现状, 提出了连接主义视域下 TPACK 框架的大学英语教学构建及实现途径。

\section{关键词一连接主义；TPACK；大学英语教学}

\section{I. 引言}

国家中长期教育改革和发展规划纲要（2010-2020 年） 提出了提高人才培养质量, 优先发展教育, 构建现代教育 体系, 建设学习型社会, 培养大批创新人才的要求。推进 教育信息化是顺应世界信息化发展趋势。当前, 信息技术 发展日新月异, 移动互联网、物联网、云计算、大数据等 技术日渐成熟, 信息化对人类的生产、生活乃至思维、学 习方式等都已产生巨大影响, 为教育改革发展注入了新活 力。

我们所处的 21 世纪发达工业时代 科技发展日新月异, 互联网、大数据、云计算、机器人等现代技术深刻改变着 人类社会, 正如拉塞尔 - L- 阿克夫(Russell L.Ackoff ), 丹尼 尔. 格林伯格(Daniel Greenberg) 指出这个时代的特征: 在
全球范围内直接获取最新知识的能力; 可在任何地方与其 他拥有共同兴趣之人进行互动等的能力; 接受对于任何文 化共享所做的及时反馈的能力; 把新思想、新发明和新艺 术创造广而告之的能力; 一生中从一种活动转向另一种活 动的等能力。现代技术发展深刻影响课堂教学, 以印刷文 本为媒介转向以声音为媒介的过渡 (Hedge,2002), 在国际 开放教育资源运动背景下, 强调以学生为中心, 利用信息 化环境下优质教育教学资源与服务技术支持开展翻转课堂、 混合学习(Blending Learning)、适时教学( Just in Time Teaching, JiTT) 等教学新模式, 师生共同进行线上线下教 学和课内课外活动。

此外, 知识更新周期缩短对高校实验教学提出了新要求 随着社会发展, 知识更新周期越来越短, 联合国教科文组 织的研究表明，信息通信技术带来了人类知识更新速度的 加速, 20 世纪 60--70 年代, 一般的知识更新周期为 5--10 年, 80 至 90 年代许多学科的知识更新周期缩短为 5 年, 而 进入 21 世纪许多学科的知识更新周期已缩短至 2 年到 3 年 （高东峰, 2018）。

马克斯・范梅南 (2001) 说: “教育需要转向体验世界。 体验可以开启我们的理解力, 恢复一种具体化的认知感。” 通过信息技术装备, 学生更能提升课堂参与度, Anderson 谈到 “使学生在一起学习的同时, 对时间、空间、活动、身 份与关系保持较多的个人控制”（Anderson，2005），便于 学生使用文字、图像、声音等多种符号资源进行信息整合 与知识建构, 具有支持个别化学习 (individualized learning) 与协作学习的潜力（Anderson,2005:Dalsgaard,2006）。

教育部 2017 年公布的大学英语教学指南明确提出大学 英语应大力推进最新信息技术与课程教学的融合, 继续发 挥现代教育技术, 特别是信息技术在外语教学中的重要作 用, 有学者指出大学英语教学长期存在重“教”轻“学”, 重“讲 授”轻“能力”, 教学上重“控制, 统一”轻“开放, 个性”, 学 习上重“接受式”轻“探究式”的五重五轻现象。传统的大学英 语教学目标失衡, 以考证为目标, 忽略学生应用能力培养: 
授课以教师为中心, 不能照顾学生个性化发展需要; 教学 工具以白板笔，书本为主，多媒体的使用也仅限于简单的 几项操作：教学课件的展示，听力材料及视频的播放，未 能有效利用多媒体技术实现课堂的翻转; 师生的交流仅限 于课堂上; 学生成绩评价多以纸质考卷结果为主, 缺少科 学合理的学习过程的评价。

为了深入了解大学生学习情况, 作者在 2018 年 10 月对 广东省某所高校在校外语系大三, 大四学生进行主题为关 于大学生课余时间安排和利用的调查, 发放 110 份调查问 卷, 回收有效问卷 100 份, 问卷内容如课余时间的安排, 学生选择比较侧重上网娱乐占了 33.78\%, 学习专业课程 $27.03 \%$ ，体育锻炼占 $20.27 \%$; 说明在校生使用电子产品, 如电脑, 手机上网时间比较多, 没有做好个人学习管理, 学会如何利用信息技术和设备进行有效学习, 而目前课堂 上教师要求学生的比较普遍的电脑技能使用方面主要是 PPT 制作, 与布置的任务有关的相片及视频的处理和优化。 课外您会看什么类型的书 $63.51 \%$ 学生选择与学习无关, 但 是自己感兴趣的书, 而与学习有关的书仅仅占了 $16.22 \%$; 说明同学们的学习积极性有待提高, 如何有效提升学生课 余时间使用率, 涉及到教师教学设计, 教学方法和手段的 提升, 如采用翻转课堂方法, 在课前布置学生利用信息技 术设施完成预习, 相关知识点学习, 拓展等工作。在课余 时间有没想到学习问题上，43.24\%学生回答经常想，可是 做不到, 36.49\%同学有想到学习并且做到了; 这说明学生 还是有积极主动学习的愿望。在认为课余时间有何影响的 问题中， 79.73\%的同学认为做自己想做的事情，包括有更 多的时间与同学交往，增进友情，有更多的时间阅读及参 加一些课外活动, 说明学生不仅注重学习效果, 同时也学 习如何与人交往, 如何进行社交活动, 丰富个人业余生活 的意愿比较强。但是, 我们从调查结果发现同学们的学习 和娱乐的时间不够合理; 学习目标不顾强, 时间使用效率 不高; 缺乏对业余时间计划和引导，比较随心所欲。同时 也反映了目前英语教学的问题, 教师没有意识引导学生进 行课外主动学习, 没有利用好现代教学信息设施和设备, 发挥他们的优势, 在教学方法和手段上没有与时俱进, 如 何激发学生的学习热情, 提升知识的主动搜索能力, 主动 解决问题的能力, 有目标有效率利用好课余时间, 面对学 情不同的学生和信息时代的变化, 更有效的提升教学质量, 引导学生是我们大学英语教师需要思考的问题。

综上所述，国内外形势发展及信息技术革新发展，传统 大学英语教学弊端对课程教学提出了更高要求, 大学英语 教学面临着巨大挑战。

从 2002 年起,教育部推动信息化技术辅助大学英语教学 模式, 但缺乏深层次研究, 以“连接主义大学英语”为主题查 阅中国知网, 基于连接主义理论的大学英语教学的文献从 2009 年到 2016 年主题和内容涉及到信息技术的仅为 8 篇。 基于连接主义理论的大学英语教学, 有学者从大学英语教 学中思辨能力培养的角度研究 (应英, 张媛, 2016) ; 有 从大学英语教材中多维信息流构建角度研究 (李霄翔, 鲍 敏, 2009); 从网络辅助教学平台在大学英语教学中的应 用 (王芳，2012:; 王华，2013; 陈芙蓉，2018）; 有从教 材知识体系研究（袁晶, 王利民, 2015）; 网络课程设计
角度研究（王岩，吴丽萍，2012）; 以“大学英语信息技术 教学”为主题进行搜索， 2007 年到 2017 年共 33 篇，核心期 刊 5 篇, 这 5 篇涉及到教师的专业发展, 教师的信息素养, 优化多媒体中心的研究角度, 而以“TPACK 框架下大学英 语教学”为主题的文献仅为两篇。以上文献研究表明国内学 者日益重视信息技术辅助大学英语教学, 但是 TPACK 框架 下大学英语教学研究比较少涉足, 从连接主义视域下 TPACK 辅助大学英语教学研究同样存在这种情况。

\section{II. 连接主义理论}

加拿大教育家 George Siemens 从 2004 年起提出一种以 网络学习为基础的学习理论一一连结主义, 连接主义有两 个核心元素：节点与连接。他认为: “我们今天的许多知识 不是存在与心智之中，而是分散在许多个体、图书馆、网 络上的各种观点和软件之中。”现时性是所有联结主义学习 活动的意图, 学习是一种创造知识而不仅仅是汲取知识的 过程, 知识也有半衰期, 学习也是可变的 (Siemens,2005), 在连接主义看来，学习活动是持续的、发展的、开放性的、 动态的, 也是多元的, 交叉性的。网络学习可以有静态节 点, 即稳定的知识结构, 也有动态节点, 即新信息的增添 和数据的不断变化，还包括“自动更新节点”，即与原信息源 紧密相连的节点, 产生高度流动性, 体现最新信息

(Siemens,2006)。黄志华 (2011) 提出建立“动态型网络”, 可设计多元型知识网络的学习模块, 以模块形式通过搜集, 分析，传递和整合商务信息及其交叉学科知识，以模块设 计的形式表达出来, 帮助学生掌握不同领域的知识点, 在 学习过程中, 发现交叉学科的知识间连接, 通过学习资源 交互设计深入学习, 培养的学生具有特色和竞争力。

连接主义改变了学习内容, 在网络创建连接后, 知识不 断更新, 学习内容不断改变; 学习者按照个人需求关注知 识相关性, 学习资源的即时性非常重要, 满足学习者学习 需求; 连接主义保证学习内容的现时性, 在教学过程中, 提升学习效应, 增强知识的流通, 需要系统管理, 互联网 搜索引擎等先进的教育现代化技术装备作保障; 电脑和手 机的普及, 教学软件的层出不穷让学习者随时随地可以与 知识内容连接, 保证了学习者终身学习; 外部知识的复杂 及更新速度的加快, 学习者通过信息技术运用及学习平台 的创建, 按类建立知识的储存与所需知识的各种节点, 无 形中知识者的学习行为迁移到网络上, 正面加强了学习者 连接各种不同领域, 概念, 知识内容的能力; 外部因素 (社 会, 社区, 同学等) 同样影响学习。

\section{III. 连接主义视域下的大学英语信息技术化教学实现}

\section{A. 连接主义基础上 TPACK 框架的大学英语教学构建}

大学英语课堂是由师生, 教学环境等构成的整体, 借助 先进的教育技术装备之力, 通过教学实现智能信息流动。

TPACK 全称为整合技术的学科教学知识(Technological Pedagogical and Content Knowledge), Mishara 和 Koehler (2005) 在学科教学知识 (PCK) 基础上提出整合技术的学 科教学知识 (TPACK) , 第一次从理论上把技术知识融入 教师知识体系中。TPACK 的核心有: 学科知识 (CK)、技 术知识（TK）、教学法知识（PK）。在三者基础上, 又形 
成了 PCK(学科教学法知识)（TPK) 整合技术教学法知识、

(TCK) 整合技术的学科知识, Mishara 和 Koehler (2006) 认为专家型教师具备完善的技术知识、学科知识和教学法 知识, 可以根据教学情境整合 PCK,TPK,TRACK 教学。詹 艺和任友群(2010:78-87)指出 TPACK 自身概念, TPACK 与 教师教育, K12 课堂技术整合, 实践的研究。

王琦 (2014) 在 2014 年对 150 名外语教师的 TPACK 结 构及其技术整合自我效能研究进行了研究, 发现外语教师 信息技术知识水平不高, 整合技术的信心不足, 教师 TPACK 知识结构认知水平有待提升, 技术整合自我效能及 各维度水平中等, TPACK 与技术整合自我效能呈显著正相 关, 可以提升教学信心, 对教师技术整合的自我效能有显 著影响。因此, 我们需更关注大学英语教师的 TPACK 结 构提升, 这对学校建设工作, 专业建设工作及外语人才培 养的提升工作大有裨益。

在 TPACK 基础上, 大学英语教学可借助信息技术装备, 整合课程教学与信息技术, 实现专业知识与信息技术高度 融合。教师执教信念和专业知识良性互动可以有力的推动 教师自身的技术与教学发展, 促进大学英语教师 TPACK 的 发展。

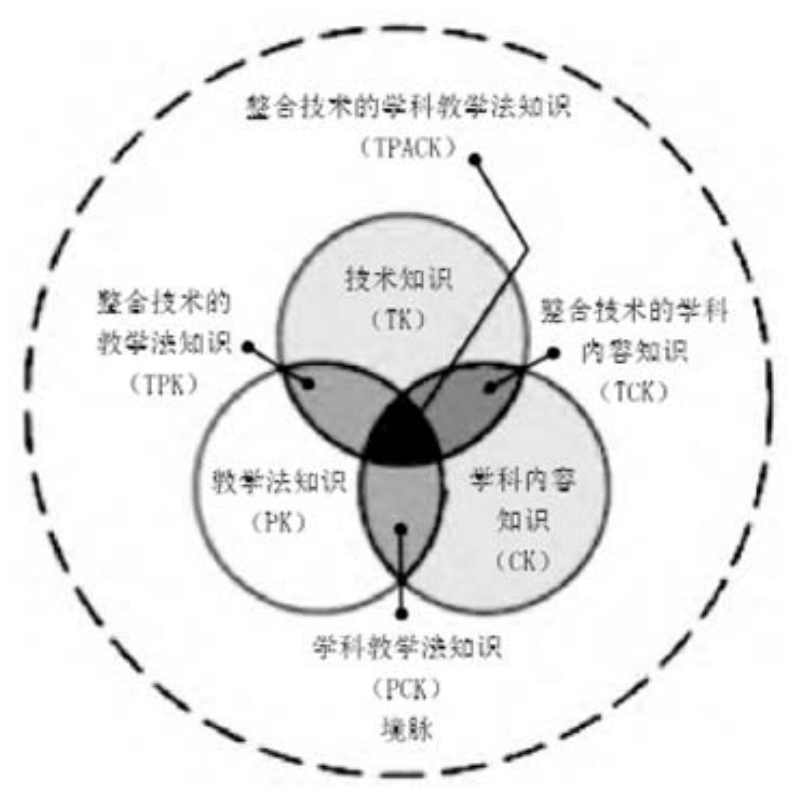

图 I TPACK 框架新版韦恩示意图（詹艺・任友群・2010:79）

\section{B. 构建多模块课程群}

构建大学英语课程群, 各模块间相辅相成, 实现学生各 种能力均衡发展, 见表 1 。
表 I 大学英语课程体系模块

\begin{tabular}{|l|l|l|}
\hline \multicolumn{1}{|c|}{ 模块 } & \multicolumn{1}{|c|}{ 课程群 } & \multicolumn{1}{c|}{ 功效 } \\
\hline $\begin{array}{l}\text { 通用英语课程 } \\
\text { 模块 }\end{array}$ & $\begin{array}{l}\text { 大学英语、听力、口 } \\
\text { 语、写作、阅读、翻译 } \\
\text { 等 }\end{array}$ & 为学生打下坚实语言基础 \\
\hline $\begin{array}{l}\text { 跨文化交际课 } \\
\text { 程模块 }\end{array}$ & $\begin{array}{l}\text { 英美概况、跨文化交际 } \\
\text { 等 }\end{array}$ & $\begin{array}{l}\text { 培养学生的跨文化意识, 提 } \\
\text { 升学生文化差异认知水平 }\end{array}$ \\
\hline 运用工具模块 & 计算机运用 & $\begin{array}{l}\text { 使学生学会利用教育信息 } \\
\text { 识术设备, 主动获取学科知 }\end{array}$ \\
\hline $\begin{array}{l}\text { 专门用途英语 } \\
\text { 课程模块 }\end{array}$ & $\begin{array}{l}\text { 学术英语、职业英语 } \\
\text { 增强学生运用英语进行专 } \\
\text { 业和学术交流, 从事工作的 } \\
\text { 能力 }\end{array}$ \\
\hline
\end{tabular}

C. 搭建共享的多元化, 开放性课程平台

构建大学英语网络教学平台、多媒体技能训练平台、外 语资源服务平台、多媒体素材编辑平台等, 实现分层管理、 远程监控、智能交互。

学校为师生提供先进的教育技术装备及无障碍的共享平 台, 定期开展针对教师教育技术技能, 教学能力提升的教 学观摩, 培训等活动。创建社会服务平台, 师生投入区域 经济服务工作，与当地企业、行业协会共同打造绿色无障 碍三位一体的“生态链”, 形成彼此依存, 互利共生的良性关 系, 通过校企合作平台, 及时了解人才培养需求, 动态调 整大学英语教学, 两条腿走路, “引进来”, 引进校外专家, 通过讲座, 授课, 沙龙等形式传递行业信息, 分享工作心 得和技能; “走出去”, 通过教师的顶岗实践, 学生的校外实 训形式参与企业一线工作, 通过社交软件 (博客, 维基等) 共享, 聚合的特点, 形成学习者、教师、专家构成的英语 数字素养网络学习共同体。

\section{D. 拓深师生信息技术素养及技能}

教育家陶行知积极推行“教学做合一”的教育理念, ”做一 个现代人必须取得现代的知识, 学会现代的技能, 感觉现 代的问题，并以现代化的方法发挥我们的力量“（1986，第 3 卷： 248 页）。

教师个人理念上, 由于教师是教学的设计者、引导者, 教师个人执教理念也需更新, 学习, 教师利用通过社交软 件 (博客, 维基等) 共享, 聚合的特点, 形成学习者、教 师、专家构成的英语数字素养网络学习共同体。

教学实施上, 教师应鼓励传统课堂教学与在线教育, 结 合混合学习、协作学习、案例学习、沉浸学习、移动学习 教学模式的融合, 实现线上线下课堂内外优势互补, 面向 过程和能力的教学评价 (李红美、陆国栋, 张剑平, 2015), 实践课程教学以 PBL(Problem-Based Learning) 教学法, 以 问题激发学生学习的动力从而引导学生把握学习内容的教 学方法, 促进学生自学, 掌握职业工作环节技能。依据建构 主义的学习理论, 构建网络教学模块与基于社会性软件的 网络学习社区组合而成的英语数字素养学习社区, 提供自 主、开放的学习、交流、协作与反思平台, 改变传统的课 
本驱动式的大学英语教学模式, 促使学生使用多种符号资 源积极构建个性化的学习者身份, 增强学生元认知能力。

教学设计上, 融入搜索, 评析, 整合数字信息及运用文 字、图像、声音等多种符号资源进行学术交流的能力的培 养, 强调评判性与创造性思维能力, 拓展以学习语言知识 为主的传统教学内容。构建网络教学模块与学习社区组合 而成的英语数字素养学习社区, 为学术开展项目活动与评 价活动提供学习交流平台, 改变传统的课本驱动式的大学 英语教学模式, 促使学生使用多种符号资源构建个性化的 学习者身份, 增强学生元认知能力。

个人信息能力上, 教师需掌握课堂相关的软件工具, 升 级个人信息技术装备运用技术，如学习运用课程管理系统 ( MediaCore Video Learning Platform、eFront Learning、 Edmodo. Flipped Lessons. Blackboard. Moodle. Sakai 等) 、 课件制作工具( Articulate Storyline、Lectora、OutStart Trainer、 Composica 等)，PowerPoint 插件工具 (Wondershare、 Ispring、Microsoft Producer) 、屏幕录像软件( Camtasia Studio、Jing、Knovio、Present. Me、Screen Flow 等), 内容展示工具( Prezi、Slideshare 等)。

教学引导上, 学生不再是语言知识被动的接受者, 信息 技术的辅助和教师引导下, 通过感知, 体验, 参与, 实践, 协作, 实现大学英语教学学习目标, 利用丰富的教学资源 和信息技术装备, 拓展学习和实践渠道, 主动参与课程资 源的开发和利用, 在课堂教学中提升个人的心智, 语言与 信息技术运用综合等能力。

\section{E. 创立动态多元化评价体系}

采用评价表与评价量规相结合, 为研究过程与结果提供 了稳定, 有效, 适时的反馈, 拓展目前大学英语教育中较 为单一的, 基于测量范式的评价传统。参照 Doing English Digital 评价模式的设计方案, 杨凤 (2008) 结合北京工业 大学的大学英语课程, 针对学生特点设计评价任务要求学 生结合大学英语新视野教材内容, 通过互联网及数据库等 数字资源搜集相关信息进行研究, 完成研究报告并使用以 “content, clarity ,critical/creative thinking"为评价维度的量规 进行评定, 取得了一定效果, 促使学生学习的同时, 有清 晰的维度衡量学习结果, 不仅有专业知识吸收评价, 还有 展现沟通能力, 思辨能力的评价, 对于学生的思辨能力及 学习能力提升提供了有效的途径。

\section{结语}

教育信息技术深刻改变着高校课堂教育, 同样影响着大 学英语教学的教育理念, 教学手段和教学模式, 基于连接 主义理论 TPACK 框架的大学英语教学强调学科知识, 技术 知识与教学法知识的高度融合, 为大学英语教学资源的选 择, 信息技术手段的实施, 平台的选择, 课堂教学的设计 和教学评估方法提供了新的思路和方法,顺应素质教育强调 发展学生应用能力, 对教师专业化发展影响深远, 促进了 教师执教信心，也为课程改革指明了方向。

\section{致谢}

本文是广东省哲学社会科学研究外语学科专项重点项目 “国家标准指导下的外语专业人才思辨能力培养研究”( 立 项编号 GD16WXZ20)的阶段性成果之一。

\section{REFERENCES}

[1] Anderson, T. (2005)Distance learning---Social software's killer ap? Retrieved November $\quad 12, \quad 2007$ from http://www.unisa.edu.au/odlaaconference/PPDF2s/13\%20odlaa\%20-\%2 0 Anderson.pdf

[2] Dalsgaard,C.(2006).Social software: E-learning beyond learning management systems.European Journal of Open, Distance and E-learning. Retrieved January 5, 2007 from http://www.eurodl.org/materials/contrib/2006/Christian Dalsgarrd.htm

[3] Hedge, T. 2002. Teaching and learning in the language classroom [M].Shanghai: Shanghai Foreign Language Education Press.

[4] Koehler, M.J. and P.Mishra.2005. What happens when teachers design educational technology? The development of technological pedagogical content knowledge [J].Journal of educational computing research,32(2):131-52.

[5] Koehler, M.J. and P.Mishra.2005.What happens when teachers design educational technology? The development of technological pedagogical content knowledge [J].Journal of educational computing research,32(2):131-52.

[6] George Siemens, Connectivism: A learning Theory for the Digital Age. Elearningspace http://www.elearnspace.org/Articles/connectivism.htm,2004.

[7] Max Van Manen. Educational Wisdom - Implications of Educational Wisdom[M].,Educational Science Press.2001:269

[8] The Thirteenth Five-Year Plan for the Development of National Education, Ministry of Education of the People's Republic of China, http://www.moe.gov.cn/jyb_xxgk/moe_1777/moe_1778/201701/t20170 119 295319.html

[9] Ministry of Education of the People's Republic of China, http://www.moe.gov.cn/jyb_xxgk/moe_1777/moe_1778/201701/t20170 119_295319.html

[10] Gao Dongfeng. Requirements, Idea and Path of Experimental Teaching Reform in Colleges and Universities in the Information Age [J], Higher education research2018.(4):93-96

[11] Zhan Yi, Ren Youqun. The connotation of subject Teaching method knowledge of Integrated Technology and its Research status quotation[J].Journal of Distance Education,2010(4)

[12] Tao Xingzhi.Tao Xingzhi complete works[M].Hunan Education Press, vol. 3: 248

[13] Deng $\mathrm{Li}, \mathrm{Hu}$ Ling. Exploring the knowledge system of excellent Teachers in College English Teaching based on TPACK Model[D].Hunan University, Foreign Linguistics and Applied Linguistics.

[14] Li Xingzhi.The opening report of "the History of the Development of Educational Technology and equipment in China"[J].Chinese Educational Technology Equipment,2012(8):8.

[15] Alan. History of Educational equipment Theory in China,Capital Normal University Press,2016:20.

[16] Ying, Zhang Yuan. A study on the cultivation of speculative ability in College English Teaching based on Connectivism [J]. Journal of Chifeng College (philosophy and Social Sciences in Chinese) 2016 (37) 09148-149.

[17] Li Xiaoxiang, Bao Min. A study on the Construction of Multi-dimensional Information flow in College English textbooks: a Connectionist and experiential philosophy Perspective [J]. Chinese foreign languages 2009 (31) 82-88.

[18] Chen Furong. The Application of Network-based Learning platform in College English Teaching [J]. Journal of Mudanjiang University No., 2018, (27) 03:139-141.

[19] Yuan Jing, Wang Limin. A Study on the Knowledge System of College English Textbooks for Art and Physical Education from the Perspective 
of Connectivism [J]. A study of higher Education in Heilongjiang Province.2015, 03, 174-176.

[20] Wang Yan, Wu Liping. Connectionist College English Web-based Curriculum Design [J]. Journal of Sichuan Institute of Education.2012, 28(06)85-88.

[21] Li Mingxi. Research on the Construction of Cloud Space and Teaching Application in College English Learning based on TPACK [J]. China's audio-visual education, 2018(5)53-60.

[22] Dengdi,A Review on the Application of flipping classroom Model in College English Teaching [J].Foreign Language World,2016,(4):89-96.

[23] Zhang Fengjuan, Lin Juan, et al. A study on TPACK characteristics and Development of College English Teachers [J]. China Audio-visual Education: 2015, (5): 124-129.

[24] Wang Qi, Study on Foreign Language Teachers'TPACK Structure and the Technology Integration with Self-efficacy [J]. Computer-assisted Foreign language Education 2014,7(158):14-20 